\title{
Stabilization of internal space in noncommutative multidimensional cosmology
}

\author{
N. Khosravi ${ }^{1 *}$, S. Jalalzadeh ${ }^{1 \dagger}$ and H. R. Sepangi ${ }^{1,2 \ddagger}$ \\ ${ }^{1}$ Department of Physics, Shahid Beheshti University, Evin, Tehran 19839, Iran \\ ${ }^{2}$ Institute for Studies in Theoretical Physics and Mathematics, P.O. Box 19395-5746, Tehran, Iran
}

January 2, 2018

\begin{abstract}
We study the cosmological aspects of a noncommutative, multidimensional universe where the matter source is assumed to be a scalar field which does not commute with the internal scale factor. We show that such noncommutativity results in the internal dimensions being stabilized.

PACS: 04.20.-q, 04.50.+h
\end{abstract}

\section{Introduction}

Multidimensional theories of general relativity and cosmology are an active area of research and have become even more so in the past few decades. As a key reason for this interest one may point to the common roots which such theories have with string theory [1] and its generalization, the Mtheory [2]. Also, their success in describing the mass hierarchy problem [3], the cosmological constant problem [4] and unification of interactions have been notable in the recent past. However, as our observed universe seems to have only four dimensions, at least at energies below the unification scale, multidimensional theories face the challenging task of dealing with these internal dimensions, that is, making them appropriately small and stable. A myriad of explanations and solutions to these problems have been suggested since the birth of such theories $[5,6]$.

One approach in this regard is that of the introduction of noncommutativity into the theory. However, one should be careful to distinguish noncommutativity in the geometry of space-time from that between the fields. Snyder did the first work on noncommutative space-time [7] which stirred a large amount of interest $[8,9,10]$. This interest has some roots in string and M-theories [11, 12]. These noncommutative models have been able to offer interesting results in dealing with problems such as IR/UV mixing and non-locality [13], Lorentz violation [14] and new physics at very short distance scales [15]. A different approach is the introduction of noncommutativity between the fields [16]. Noncommutative cosmology $[17,18]$ is an example of such an approach and has benefited from it greatly in that it can offer a view of the semiclassical approximation of quantum gravity and may be used in tackling the cosmological constant problem [19]. Also, this kind of noncommutativity is used in [20] to address the stabilization of internal dimensions and the cosmological constant problem.

In this paper, we have considered a multidimensional cosmology with two scale factors for external and internal spaces in the presence of a matter field. We show that if the matter field does not commute with the scale factor associated with the internal dimensions, an interesting result, namely

*email: n-khosravi@sbu.ac.ir

${ }^{\dagger}$ email: s-jalalzadeh@sbu.ac.ir

${ }^{\ddagger}$ email: hr-sepangi@sbu.ac.ir 
stabilization of the internal dimensions emerges. In our approach, in contrast to other approaches $[5,21,22,23]$, the existence of a potential term with specific properties corresponding to the scalar field is unnecessary. It will be shown that noncommutativity plays a crucial role in the study of stabilization of the internal dimensions. It should however be remarked that the model presented here is a toy model in that in a more comprehensive setting, one should consider a more general metric for which the brackets of all the components with the scalar field should be calculated and taken into account.

\section{The Model}

Consider a cosmological model on a manifold defined as

$$
M=R \times M_{1} \times M_{2},
$$

with a metric of the form

$$
g=-e^{2 \gamma(\tau)} d \tau \otimes d \tau+e^{2 u(\tau)} d x^{i} \otimes d x^{i}+e^{2 v(\tau)} d x^{\alpha} \otimes d x^{\alpha},
$$

where $i=1,2,3$ and $\alpha=1,2, \ldots, d$ with $d$ being the dimension of the internal space. Note that the above homogeneous metric is also isotropic with different scale factors with respect to the internal and ordinary spaces separately. These models are natural multidimensional generalizations of the Friedmann as well as Kasner universes [1]. Here, we investigate a model with a cosmological constant $\Lambda$ and a non-interacting homogenous minimally coupled scalar field $\phi$ represented by the potential $U(\phi)$. The action functional can be written as

$$
\mathcal{S}=\frac{1}{2 \kappa^{2}} \int d^{D} x \sqrt{g}\{R[g]-2 \Lambda\}+\mathcal{S}_{\phi}+\mathcal{S}_{Y G H},
$$

where $R[g]$ is the scalar curvature of the metric (2), $\kappa^{2}$ is the $D$-dimensional gravitational constant, where $D=d+3$ and $\mathcal{S}_{Y G H}$ is the usual York-Gibbons-Hawking boundary term. The action of the matter field $\mathcal{S}_{\phi}$ is

$$
\mathcal{S}_{\phi}=\int d^{D} x \sqrt{g}\left[-\frac{1}{2} g^{\mu \nu} \partial_{\mu} \phi \partial_{\nu} \phi-U(\phi)\right]
$$

Assuming that all fields are functions of $\tau$ and using metric (2), action (3) can be written as

$$
\mathcal{S}=k \int d \tau L
$$

where $k$ is a constant and

$$
L=-\frac{1}{2} e^{-\gamma+\gamma_{0}}\left[6 \dot{u}^{2}+\left(d^{2}-d\right) \dot{v}^{2}+6 d \dot{u} \dot{v}-\kappa^{2} \dot{\phi}^{2}\right]-e^{\gamma+\gamma_{0}}\left[\kappa^{2} U(\phi)+\Lambda\right] .
$$

Here, a dot represents differentiation with respect to $\tau$ and $\gamma_{0}=3 u+d v$. Choice of the harmonic time gauge as $\gamma=\gamma_{0}[24]$ results in

$$
L=-\frac{1}{2}\left[6 \dot{u}^{2}+\left(d^{2}-d\right) \dot{v}^{2}+6 d \dot{u} \dot{v}-\kappa^{2} \dot{\phi}^{2}\right]-e^{2(3 u+d v)}\left[\kappa^{2} U(\phi)+\Lambda\right] .
$$

For the above Lagrangian one may write the corresponding Hamiltonian as

$$
H=\alpha p_{u}^{2}+\beta p_{v}^{2}+\delta p_{u} p_{v}+\frac{1}{2 \kappa^{2}} p_{\phi}^{2}+e^{2(3 u+d v)}\left(\kappa^{2} U(\phi)+\Lambda\right)
$$


where $p_{u}, p_{v}$ and $p_{\phi}$ are the momenta conjugate to $u, v$, and $\phi$ respectively and $\alpha, \beta$, and $\delta$ are the following constants

$$
\begin{aligned}
\alpha & =\frac{d-1}{6(d+2)}, \\
\beta & =\frac{1}{d(d+2)}, \\
\delta & =\frac{-1}{d+2} .
\end{aligned}
$$

Equations of motion correspond to Hamiltonian (8) become

$$
\begin{aligned}
\dot{u} & =\{u, H\}_{P}=2 \alpha p_{u}+\delta p_{v}, \\
\dot{p_{u}} & =\left\{p_{u}, H\right\}_{P}=-6 V, \\
\dot{v} & =\{v, H\}_{P}=2 \beta p_{v}+\delta p_{u}, \\
\dot{p_{v}} & =\left\{p_{v}, H\right\}_{P}=-2 d V \\
\dot{\phi} & =\{\phi, H\}_{P}=\frac{1}{\kappa^{2}} p_{\phi}, \\
\dot{p_{\phi}} & =\left\{p_{\phi}, H\right\}_{P}=-\kappa^{2} e^{2(3 u+d v)} \frac{d U(\phi)}{d \phi},
\end{aligned}
$$

where $V=e^{2(3 u+d v)}\left(\kappa^{2} U(\phi)+\Lambda\right)$ is the effective potential. Now, using equations (10), we obtain

$$
\begin{aligned}
\ddot{u} & =-2(6 \alpha+d \delta) V, \\
\ddot{v} & =-2(2 d \beta+3 \delta) V, \\
\ddot{\phi} & =-e^{2(3 u+d v)} \frac{d U(\phi)}{d \phi} .
\end{aligned}
$$

To address the stabilization problem one usually assumes the existence of a potential $U(\phi)$ with a minimum at a definite value. In this mechanism, in the neighborhood of the minimum, stabilization of the extra dimensions for Ricci-flat [21] and non-flat $[5,22,23]$ internal spaces can be achieved. This mechanism does not work in our model, the reason being the special gauge chosen here. To be more specific, if we use the mechanism used in [21], the resulting solutions obtained from equations (11) may be written as

$$
\begin{aligned}
u & =b_{1} \tau+b_{2}, \\
v & =b_{3} \tau+b_{4}, \\
\phi & =b_{5} \tau+b_{6},
\end{aligned}
$$

from which one can easily write the scale factors as

$$
\begin{array}{r}
R(\tau)=e^{2 u(\tau)}=e^{2\left(b_{1} \tau+b_{2}\right)}, \\
a(\tau)=e^{2 v(\tau)}=e^{2\left(b_{3} \tau+b_{4}\right)} .
\end{array}
$$

It is obvious that the scale factors diverge or converge according to the sign of $b_{1}$ and $b_{3}$, and there would no stabilization for the internal dimensions. In the next section we introduce the notion of noncommutativity and show that in the harmonic time gauge, it enables us to address the question of stabilization.

\section{Noncommutative solutions}

Let us now concentrate on the study of noncommutativity concepts with Moyal product in phase space. The Moyal product may be traced to an early intuition by Wigner [25] which has been 
developing over the past decades [26]. Noncommutativity in classical physics [27] is described by the Moyal product law between two arbitrary functions of position and momenta

$$
\left(f \star_{\alpha} g\right)(x)=\left.\exp \left[\frac{1}{2} \alpha^{a b} \partial_{a}^{(1)} \partial_{b}^{(2)}\right] f\left(x_{1}\right) g\left(x_{2}\right)\right|_{x_{1}=x_{2}=x},
$$

such that

$$
\alpha_{a b}=\left(\begin{array}{cc}
\xi_{i j} & \delta_{i j}+\sigma_{i j} \\
-\delta_{i j}-\sigma_{i j} & \zeta_{i j}
\end{array}\right)
$$

where $N \times N$ matrices $\xi$ and $\zeta$ are assumed to be antisymmetric. Also, $2 N$ is dimension of the classical phase space $\left(x_{i}, p_{i}\right)$ and $i=1,2, \ldots, N$. With this product law, deformed Poisson brackets can be written as

$$
\{f, g\}_{\alpha}=f \star_{\alpha} g-g \star_{\alpha} f
$$

where

$$
\begin{aligned}
\left\{x_{i}, x_{j}\right\}_{\alpha} & =\xi_{i j} \\
\left\{x_{i}, p_{j}\right\}_{\alpha} & =\delta_{i j}+\sigma_{i j} \\
\left\{p_{i}, p_{j}\right\}_{\alpha} & =\zeta_{i j} .
\end{aligned}
$$

It is worth noting at this stage that in addition to noncommutativity in $\left(x_{i}, x_{j}\right)$ we have also considered noncommutativity in the corresponding momenta. This should be interesting since its existence is in fact due essentially to the existence of noncommutativity on the space sector $[26,27]$ and it would somehow be natural to include it in our considerations. Now, consider the following transformations in the classical phase space $\left(x_{i}, p_{i}\right)$

$$
\left\{\begin{array}{l}
x_{i}^{\prime}=x_{i}-\frac{1}{2} \xi_{i j} p_{j}, \\
p_{i}^{\prime}=p_{i}+\frac{1}{2} \zeta_{i j} x_{j} .
\end{array}\right.
$$

It can easily be checked that if $\left(x_{i}, p_{i}\right)$ obey the usual Poisson algebra, then

$$
\begin{aligned}
& \left\{x_{i}^{\prime}, x_{j}^{\prime}\right\}_{P}=\xi_{i j} \\
& \left\{x_{i}^{\prime}, p_{j}^{\prime}\right\}_{P}=\delta_{i j}+\sigma_{i j} \\
& \left\{p_{i}^{\prime}, p_{j}^{\prime}\right\}_{P}=\zeta_{i j},
\end{aligned}
$$

where $\sigma_{i j}$ can be written as a combination of $\xi_{i j}$ and $\zeta_{i j}$. These commutation relations are the same as those in (18). Thus, for introducing noncommutativity, it is more convenient to work with Poisson brackets (20) than the $\alpha$-star deformed Poisson brackets (18). In the noncommutative case we only assume that the scale factor associated with the internal dimensions does not commute with the matter field and keep other commutation relations unchanged as in the previous section. Therefore, the relations that change are

$$
\left\{\begin{array}{l}
\left\{v^{\prime}, \phi^{\prime}\right\}_{P}=\xi \\
\left\{p_{v}^{\prime}, p_{\phi}^{\prime}\right\}_{P}=\zeta
\end{array}\right.
$$

The Hamiltonian $H^{\prime}$ can be written as

$$
H^{\prime}=\alpha p_{u}^{\prime 2}+\beta p_{v}^{\prime 2}+\delta p_{u}^{\prime} p_{v}^{\prime}+\frac{1}{2 \kappa^{2}} p_{\phi}^{\prime 2}+e^{2\left(3 u^{\prime}+d v^{\prime}\right)}\left[\kappa^{2} U\left(\phi^{\prime}\right)+\Lambda\right]
$$


where a prime denotes noncommutative variables. With the help of transformations

$$
\begin{aligned}
& v^{\prime}=v-\frac{\xi}{2} p_{\phi}, \\
& \phi^{\prime}=\phi+\frac{\xi}{2} p_{v}, \\
& p_{v}^{\prime}=p_{v}+\frac{\zeta}{2} \phi, \\
& p_{\phi}^{\prime}=p_{\phi}-\frac{\zeta}{2} v,
\end{aligned}
$$

we can write the Hamiltonian without the prime variables, that is, those that satisfy the usual commutation relations

$$
\begin{aligned}
H & =\alpha p_{u}^{2}+\beta p_{v}^{2}+\delta p_{u} p_{v}+\frac{1}{2 \kappa^{2}} p_{\phi}^{2}+\beta \zeta \phi p_{v}+\frac{\delta \zeta}{2} \phi p_{u}-\frac{\zeta}{2 \kappa^{2}} v p_{\phi}+\frac{\beta \zeta^{2}}{4} \phi^{2}+\frac{\zeta^{2}}{8 \kappa^{2}} v^{2} \\
& +e^{2(3 u+d v)} e^{-\xi d p_{\phi}}\left[\kappa^{2} U\left(\phi+\frac{\xi}{2} p_{v}\right)+\Lambda\right] .
\end{aligned}
$$

The equations of motion can now be written easily with respect to Hamiltonian (24)

$$
\begin{aligned}
& \dot{u}=\{u, H\}_{P}=2 \alpha p_{u}+\delta p_{v}+\frac{\delta \zeta}{2} \phi \\
& \dot{v}=\{v, H\}_{P}=2 \beta p_{v}+\delta p_{u}+\beta \zeta \phi+e^{2(3 u+d v)} e^{-\xi d p_{\phi}} \kappa^{2} \frac{\partial U\left(\phi+\frac{\xi}{2} p_{v}\right)}{\partial p_{v}} \\
& \dot{\phi}=\{\phi, H\}_{P}=\frac{1}{\kappa^{2}} p_{\phi}-\frac{\zeta}{2 \kappa^{2}} v-\xi d e^{2(3 u+d v)} e^{-\xi d p_{\phi}}\left[\kappa^{2} U\left(\phi+\frac{\xi}{2} p_{v}\right)+\Lambda\right] \\
& \dot{p_{u}}=\left\{p_{u}, H\right\}_{P}=-6 e^{2(3 u+d v)} e^{-\xi d p_{\phi}}\left[\kappa^{2} U\left(\phi+\frac{\xi}{2} p_{v}\right)+\Lambda\right] \\
& \dot{p_{v}}=\left\{p_{v}, H\right\}_{P}=\frac{\zeta}{2 \kappa^{2}} p_{\phi}-\frac{\zeta^{2}}{4 \kappa^{2}} v-2 d e^{2(3 u+d v)} e^{-\xi d p_{\phi}}\left[\kappa^{2} U\left(\phi+\frac{\xi}{2} p_{v}\right)+\Lambda\right], \\
& \dot{p_{\phi}}=\left\{p_{\phi}, H\right\}_{P}=-\beta \zeta p_{v}-\frac{\delta \zeta}{2} p_{u}-\frac{\beta \zeta^{2}}{2} \phi-\kappa^{2} e^{2(3 u+d v)} e^{-\xi d p_{\phi}} \frac{\partial U\left(\phi+\frac{\xi}{2} p_{v}\right)}{\partial \phi} .
\end{aligned}
$$

To proceed any further, we expand the potential about its minimum taken at $-\Lambda / \kappa^{2}$, obtaining

$$
\begin{aligned}
\ddot{u} & =\delta \zeta \dot{\phi}, \\
\ddot{v} & =2 \beta \zeta \dot{\phi}, \\
\ddot{\phi} & =-\frac{\zeta}{\kappa^{2}} \dot{v}
\end{aligned}
$$

whose solutions are

$$
\begin{aligned}
\phi & =\frac{c_{1}}{\omega} \sin (\omega \tau)-\frac{c_{2}}{\omega} \cos (\omega \tau)+c_{3}, \\
u & =-\frac{c_{1}}{\omega^{2}} \delta \zeta \cos (\omega \tau)-\frac{c_{2}}{\omega^{2}} \delta \zeta \sin (\omega \tau)+\frac{1}{2} k \tau+c_{4}, \\
v & =-\frac{c_{1}}{\omega^{2}} 2 \beta \zeta \cos (\omega \tau)-\frac{c_{2}}{\omega^{2}} 2 \beta \zeta \sin (\omega \tau)+c_{5},
\end{aligned}
$$

where $c_{1}, c_{2}, c_{3}, c_{4}, c_{5}$ and $k$ are integration constants and

$$
\omega^{2}=\frac{2 \beta \zeta^{2}}{\kappa^{2}} .
$$

Comparison between these solutions and those presented in (12) reveals the existence of oscillating terms and a linear term in $\tau$ which only appears in the scale factor $u$. It is worth noting that even if there was no effective potential $V$ present, the above discussions and results would still hold.

Let us now study the behavior of these solutions. For simplicity we take $c_{1}=0$ so that the scale factor of internal dimensions can be written as

$$
a(\tau)=e^{2 v(\tau)}=e^{2 c_{5}} \exp \left[-\frac{4 c_{2}}{\omega^{2}} \beta \zeta \sin (\omega \tau)\right] .
$$




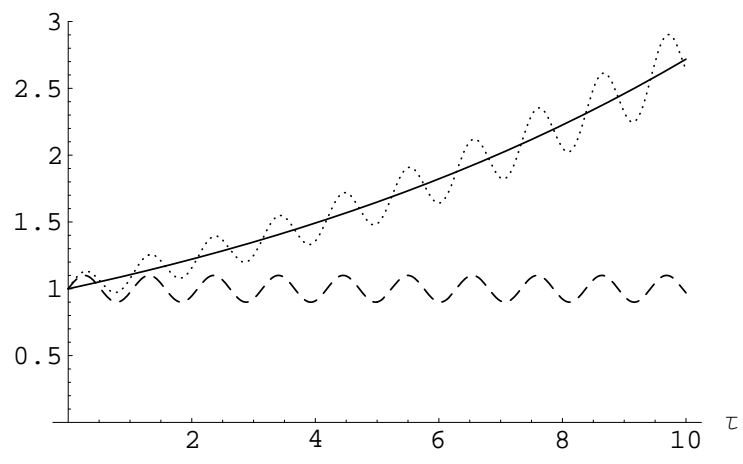

Figure 1: Commutative scale factors, solid line, ordinary noncommutative scale factor, dotted line and noncommutative scale factor associated with the internal dimensions, dashed line. Note that the solid line represents both scale factors in the commutative case.

This means that the scale factor of the internal dimensions is stabilized around $e^{2 c_{5}}$. On the other hand, the other scale factor reads

$$
R(\tau)=e^{2 u(\tau)}=e^{2 c_{4}} \exp \left[-\frac{2 c_{2}}{\omega^{2}} \delta \zeta \sin (\omega \tau)\right] e^{k \tau}
$$

The interpretation of equation (30) now depends on the sign of $k$. To have an understanding of this solution let us look at the Hubble constant $\mathrm{H}$ for the scale factor of ordinary universe in noncommutative case where it can be written as

$$
\mathrm{H}=\frac{\dot{R}}{R}=k-2 c_{2} \frac{\delta \zeta}{\omega} \cos (\omega \tau)
$$

The average of the Hubble constant (31) during its period is equal to $k$. Since observations show that the Hubble constant is positive, a positive sign for $k$ in equation (30) would also seem appropriate and natural. Having taken a positive value for $k$, one would immediately see that equation (30) would result in a large value for the scale factor of the universe consistent with present observations. This equation also shows that the usual scale factor has an oscillatory behavior and that the amplitude of oscillations grows exponentially. The behavior of the scale factors can be seen in figure 1 . It should be noted that in our noncommutative model, there is no requirement for the existence of a potential with a minimum, necessary to describe stabilization in other models $[5,21,22,23]$. Therefore, stabilization seems to have a somewhat deeper roots in noncommutativity than in the potential. In other words, the role of such a potential is played by the term $\frac{\beta \zeta^{2}}{4} \phi^{2}+\frac{\zeta^{2}}{8 \kappa^{2}} v^{2}$, appearing spontaneously in (24) and is due to the introduction of noncommutativity. This term has a minimum at $\phi_{0}=0$ and $v_{0}=0$ where upon the stabilization conditions discussed in $[5,21,22,23]$ are satisfied.

Recent observations seem to suggest that the universe has two distinct decelerated and accelerated phases (see the first reference in [28]). To account for this behavior, models based on dark energy [28] and modified gravity [29] have been suggested. To address such questions in our simple model, let us calculate the acceleration parameter $q$

$$
q=\frac{\ddot{R} R}{\dot{R}^{2}}=1+2 c_{2} \beta \zeta \frac{\sin (\omega \tau)}{\mathrm{H}} .
$$

It should now be clear that the desired behavior, that is positive or negative values for $q$ corresponding to accelerated or decelerated phases respectively, can be obtained from the above relation by tuning the parameters. Since the existence of a time dependent term in equation (32) is a direct consequence of noncommutativity, such phases could be interpreted as the direct result of this effect. Also, equation (32) suggests a periodic phase transition, as can be seen in figure 1. 


\section{Conclusions}

In this paper, we have investigated a multidimensional cosmological model with two different scale factors in the presence of a massless scalar field. In addition, we have assumed that the scale factor of the internal space does not commute with that of the matter field. The motivation for this assumption is that in different approaches to multidimensional theories, the internal space scale factors may be considered as matter fields [30,22]. So from the viewpoint of an observer in ordinary universe, there is no distinction between matter fields and scale factors of the internal space. Hence noncommutativity between these fields seems to be a natural choice. With these assumptions, it can be seen from equations $(29,30)$ that at early times or small $\tau$, the two scale factors were in the oscillating regime and can be treated as having sizes of the same order of magnitude. However, with time increasing, because of the exponential term in (30), the ordinary space scale factor begins to move away in size from the internal dimensions scale factor. So, for large $\tau$, that is for the present epoch, the scale factor of the observed universe has grown exponentially in contrast to the scale factor of the internal dimensions which are and have been oscillatory. One may therefore speaks of the stabilization of the internal dimensions in the present epoch around a small constant radius. It should be interesting to note that the various models suggested for stabilization [5, 21, 22, 23] do not work in the harmonic time gauge whereas the notion of noncommutativity proposed in this paper points in the direction of offering a solution. In addition, it suggests a way to describe the two different phases associated with our universe mentioned above.

\section{Acknowledgement}

We would like to thank B. Vakili for a careful reading of the manuscript.

\section{References}

[1] A. Zhuk, Grav. Cosmol. 2 (1996) 319-320, gr-qc/9611011.

[2] P. Horava and E. Witten, Nucl. Phys. B475 (1996) 94-114, hep-th/9603142.

[3] N. Arkani-Hamed and M. Schmaltz, Phys. Rev. D61 (2000) 033005, hep-ph/9903417.

[4] S. Jalalzadeh and H. R. Sepangi, Class. Quantum Grav. 22 (2005) 2035-2048, gr-qc/0408004, Jian Qi Shen, gr-qc/0401077.

[5] N. Arkani-Hamed, S. Dimopoulos and J. March-Russell, Phys. Rev. D63 (2001) 064020, hepth/9809124.

[6] S. Jalalzadeh, F. Ahmadi and H. R. Sepangi, JHEP 08 (2003) 012, hep-th/0308067,

F. Darabi and H. R. Sepangi, Class. Quantum Grav. 16 (1999) 1565-1575, gr-qc/9905008.

[7] H. Snyder, Phys. Rev. 71 (1947) 38.

[8] A. Connes, Noncommutative Geometry (Academic Press 1994),

A. Connes, Noncommutative Geometry, math-qa/0011193,

A. Connes, J. Math. Phys. 41 (2000) 3832, hep-th/0003006.

[9] J. C. Varilly, An Introduction to Noncommutative Geometry, physics/9709045.

[10] M. R. Douglas and N. A. Nekrasov, Rev. Mod. Phys. 73 (2002) 977, hep-th/ 0106048.

[11] N. Seiberg and E. Witten, JHEP 09 (1999) 032, hep-th/9908142.

[12] T. Banks, W. Fischler, S. H. Shenker and L. Susskind, Phys. Rev. D 55 (1997) 5112, hepth/9610043. 
[13] S. Minwalla, M. Van Raamsdonk and N. Seiberg, JHEP 02 (2000) 020, hep-th/9912072.

[14] S. M. Carroll, J. A. Harvey, V.A. Kostelecky, C. D. Lane and T. Okamoto, Phys. Rev. Lett. 87 (2001) 141601, hep-th/0105082,

C. E. Carlson, C. D. Carone and R. F. Lebed, Phys. Lett. B549 (2002) 337, hep-ph/0209077, A. Anisimov, T. Banks, M. Dine and M. Graesser, Phys. Rev. D65 (2002) 085032, hepth/0111235.

[15] R. J. Szabo, Phys. Rep. 378 (2003) 207, hep-th/0109162,

M. R. Douglas and N. A. Nekrasov, Rev. Mod. Phys. 73 (2002) 977, hep-th/0106048.

[16] J. M. Carmona, J. L. Cortes, J. Gamboa and F. Mendez, JHEP 03 (2003) 058, hep-th/0301248, J. M. Carmona, J. L. Cortes, J. Gamboa and F. Mendez, Phys. Lett. B565 (2003) 222, hepth/0207158.

[17] H. Garcia-Compean, O. Obregon and C. Ramirez, Phys. Rev. Lett. 88 (2002) 161301, hepth/0107250,

G. D. Barbosa and N. Pinto-Neto, Phys. Rev. D70 (2004) 103512, hep-th/0407111.

[18] B. Vakili, N. Khosravi and H. R. Sepangi, Class. Quantum Grav. 24 (2007) 931, gr-qc/0701075.

[19] M. I. Beciu, gr-qc/0305077,

F. Darabi, A. Rezaei-Aghdam and A. R. Rastkar, Phys. Lett. B615 (2005) 141, gr-qc/0412089.

[20] N. Khosravi, S. Jalalzadeh and H. R. Sepangi, JHEP 01 (2006) 134, hep-th/0601116.

[21] U. Guenther and A. Zhuk, Class. Quantum Grav. 18 (2001) 1441-1460, hep-ph/0006283.

[22] U. Guenther and A. Zhuk, Phys. Rev. D61 (2000) 124001, hep-ph/0002009.

[23] S. M. Carroll, J. Geddes, M. B. Hoffman and R. M. Wald, Phys. Rev. D66 (2002) 024036, hep-th/0110149.

[24] A. Zhuk, Astron. Nachr. 316 (1995) 269, gr-qc/0205116.

[25] E. Wigner, Phys. Rev. 40 (1932) 749.

[26] A. E. F. Djemai and H. Smail, Commun. Theor. Phys. 41 (2004) 837, hep-th/0309006,

E. Gozzi and M. Reuter, Mod. Phys. Lett. A8 (1993) 15,

T. Hakioglu and A. J. Dragt, J. Phys. A (2001), quant-ph/0108081.

[27] M. Przanowski and J. Tosiek, Acta Phys. Pol. B30 (1999),

A. E. F. Djemai, hep-th/0309034.

[28] T. Padmanabhan, Phys. Rept. 380 (2003) 235-320, hep-th/0212290 and refrences there in, C. Armendariz-Picon, V. Mukhanov and P. J. Steinhardt, Phys. Rev. Lett. 85 (2000) 4438-4441, astro-ph/0004134,

R. R. Caldwell, R. Dave and P. J. Steinhardt, Phys. Rev. Lett. 80 (1998) 1582-1585, astro$\mathrm{ph} / 9708069$,

P. J. E. Peebles and B. Ratra, Rev. Mod. Phys. 75 (2003) 559-606, astro-ph/0207347.

[29] S. Nojiri and S. D. Odintsov, Phys. Lett. B631 (2005) 1-6, hep-th/0508049.

[30] U. Guenther, A. Starobinsky and A. Zhuk, Phys. Rev. D69 (2004) 044003, hep-ph/0306191, N. Arkani-Hamed, S. Dimopoulos, N. Kaloper and J. March-Russell, Nucl. Phys. B567 (2000) 189-228, hep-ph/9903224. 